University of Nebraska - Lincoln

DigitalCommons@University of Nebraska - Lincoln

US Army Research

U.S. Department of Defense

2011

Digital holographic imaging of aerosol particles in flight

Matthew J. Berg

US Army Research Laboratory, matt.berg@msstate.edu

Gorden Videen

US Army Research Laboratory

Follow this and additional works at: https://digitalcommons.unl.edu/usarmyresearch

Part of the Operations Research, Systems Engineering and Industrial Engineering Commons

Berg, Matthew J. and Videen, Gorden, "Digital holographic imaging of aerosol particles in flight" (2011). US Army Research. 109.

https://digitalcommons.unl.edu/usarmyresearch/109

This Article is brought to you for free and open access by the U.S. Department of Defense at DigitalCommons@University of Nebraska - Lincoln. It has been accepted for inclusion in US Army Research by an authorized administrator of DigitalCommons@University of Nebraska - Lincoln. 


\title{
Digital holographic imaging of aerosol particles in flight
}

\author{
Matthew J. Berg ${ }^{\mathrm{a}, \mathrm{b}, *}$, Gorden Videen ${ }^{\mathrm{a}, \mathrm{b}}$ \\ a Mississippi State University, Department of Physics E' Astronomy, Mississippi State, MS 39762, USA \\ ${ }^{\mathrm{b}}$ US Army Research Laboratory, RDRL-CIE-S, 2800 Powder Mill Road, Adelphi, MD 20783-1197, USA
}

\section{A R T I C L E I N F O}

Keywords:

Holography

Light scattering

Aerosol

Imaging

\begin{abstract}
A B S T R A C T
This work describes the design and application of an apparatus to image aerosol particles using digital holography in a flow-through, contact-free manner. Particles in an aerosol stream are illuminated by a triggered, pulsed laser and the pattern produced by the interference of this light with that scattered by the particles is recorded by a digital camera. The recorded pattern constitutes a digital hologram from which an image of the particles is computationally reconstructed using a fast Fourier transform. This imaging is validated using a cluster of ragweed pollen particles. Examples involving mineral-dust aerosols demonstrate the technique's in situ imaging capability for complex-shaped particles over a size range of roughly $15-500 \mu \mathrm{m}$ micrometers. The focusing-like character of the reconstruction process is demonstrated using a $\mathrm{NaCl}$ aerosol particle and is compared to a similar particle imaged with a conventional microscope.
\end{abstract}

(c) 2011 Elsevier Ltd. All rights reserved.

\section{Introduction}

The in situ characterization of small aerosol particles is a persistent objective in applied contexts. Examples include the determination of atmospheric aerosol composition for climate modeling and the detection of biological weapons agents for defense applications. Countless measurements and calculations of single and multiple-particle scattering patterns can be found in the literature. The overall goal of such work is to infer information relating to the particles' physical form, such as size and shape, by analyzing the angular structure of these patterns, e.g. see [1]. Unfortunately, a fundamental limitation of this approach is the absence of an unambiguous quantitative relationship between a pattern and the corresponding particle properties, i.e., the so-called inverse problem. Consequently, the inference of these properties from the

\footnotetext{
* Corresponding author at: Mississippi State University, Department of Physics \& Astronomy, Mississippi State, MS 39762, USA.

Tel.: +1 785317 3378; fax: +1 6623258898 .

E-mail address: matt.berg@msstate.edu (M.J. Berg).
}

0022-4073/\$ - see front matter ๔ 2011 Elsevier Ltd. All rights reserved. doi:10.1016/j.jqsrt.2011.01.013 patterns has proved to be very difficult in practice, except for the simplest of cases.

Ideally, one would prefer to image the particles directly, thus eliminating the complexity and ambiguity associated with interpretation of the scattering patterns. However, the typical particle size range of interest for many applications is roughly $0.1-10 \mu \mathrm{m}[1,2]$. Because of this, direct images are possible in part of this range only with high numerical-aperture (NA) optics and correspondingly small focal volumes. This typically requires collection and immobilization of particle samples, and thus, such imaging is not a practical technique for particle characterization in applications requiring high sample through-put or images of the particles in their undisturbed form, i.e., in situ images.

Holography is an alternative technique that combines useful elements of both conventional imaging and scattering. Fundamentally, this is a two-step process: First, an object is illuminated with coherent light and the intensity pattern resulting from the interference of this light with that scattered by the particle is recorded. This pattern constitutes the hologram, from which an image of the object is reconstructed. Traditionally, holograms are 
recorded with photographic film due to the film's high resolution, which is required to capture the finer features of the interference pattern. The subsequent chemical development of the film is costly and time consuming, and this greatly limits the practical utility of the technique. For this reason, charged coupled device (CCD) detectors are used to record the interference pattern digitally. The resulting so-called digital hologram can then be computationally processed, rather than chemically, to reconstruct an image of the object.

Digital holographic imaging has been demonstrated in multiple small-particle systems and across visible and X-ray wavelengths, see e.g. [3-11]. Examples of work applying holography to aerosols are scarce, and to the best of our knowledge have not yet been reported for in situ imaging of aerosol particles in the $0.1-25 \mu \mathrm{m}$ size range using visible light. This article will describe the design and implementation of an apparatus that achieves imaging of particles approximately $15-500 \mu \mathrm{m}$ in size, and has the potential to image particles as small as $4 \mu \mathrm{m}$ given further design optimization. The basic concepts involved are briefly reviewed and a validation measurement using ragweed pollen particles is presented. Saharan, Tunisian, and sodium chloride $(\mathrm{NaCl})$ aerosols are used to establish the in situ capability of the apparatus. Finally, the microscope-like focusing behavior of the image-reconstruction process is demonstrated using a single $\mathrm{NaCl}$ aerosol particle.

\section{Digital in-line holography}

The apparatus in this work is based on the so-called inline holographic configuration [3]. Here, the particle, primary optical components, and detector are all colinearly arranged. The particle is illuminated by a monochromatic spherical wave and the resulting interference pattern formed by this reference wave and the light scattered by the particle is recorded by a CCD detector. Let the source of the reference wave be located at a distance $l$ from the particle and the detector at a distance $d$. Provided that $k l$ and $k d$ are large enough to satisfy the far-field conditions of [12], both the reference and scattered waves will be transverse and spherical at the detector and can be represented entirely by their scattering amplitudes

$E^{\mathrm{ref}}(\mathbf{r})=\frac{\exp (i k r)}{r} E_{1}^{\mathrm{ref}}(\hat{\mathbf{r}}), \quad E^{\text {sca }}(\mathbf{r})=\frac{\exp (i k r)}{r} E_{1}^{\text {sca }}(\hat{\mathbf{r}})$,

respectively. Then, the intensity of the total wave across the detector's face is [3]

$I^{\text {holo }}(\mathbf{r})=\frac{c \varepsilon_{0}}{r^{2}}\left|E_{1}^{\mathrm{ref}}(\hat{\mathbf{r}})+E_{1}^{\mathrm{sca}}(\hat{\mathbf{r}})\right|^{2}$,

where $c$ and $\varepsilon_{o}$ are the vacuum speed of light and electric permittivity, respectively. Expanding Eq. (2) gives

$$
\begin{aligned}
I^{\text {holo }}(\mathbf{r})= & \frac{c \varepsilon_{0}}{r^{2}}\left\{\left|E_{1}^{\text {ref }}(\hat{\mathbf{r}})\right|^{2}+\left|E_{1}^{\text {sca }}(\hat{\mathbf{r}})\right|^{2}+\left[E_{1}^{\mathrm{ref}}(\hat{\mathbf{r}})\right]^{*} E_{1}^{\text {sca }}(\hat{\mathbf{r}})\right. \\
& \left.+\left[E_{1}^{\text {sca }}(\hat{\mathbf{r}})\right]^{*} E_{1}^{\text {ref }}(\hat{\mathbf{r}})\right\} .
\end{aligned}
$$

The quantity $c \varepsilon_{0} r^{-2}\left|E_{1}^{\mathrm{ref}}(\hat{\mathbf{r}})\right|^{2}=I^{\mathrm{ref}}(\mathbf{r})$ in Eq. (3) is the intensity across the detector when no particle is present, and hence can be considered a known quantity measured before the introduction of an aerosol sample. Subtracting this reference intensity from Eq. (3) and dividing the remaining terms by it gives

$$
\begin{aligned}
I^{\mathrm{con}}(\mathbf{r}) & =\frac{I^{\text {holo }}(\mathbf{r})-I^{\mathrm{ref}}(\mathbf{r})}{I^{\mathrm{ref}}(\mathbf{r})} \\
& =\frac{\left|E_{1}^{\mathrm{sca}}(\hat{\mathbf{r}})\right|^{2}}{\left|E_{1}^{\text {ref }}(\hat{\mathbf{r}})\right|^{2}}+\frac{\left[E_{1}^{\mathrm{ref}}(\hat{\mathbf{r}})\right]^{*} E_{1}^{\text {sca }}(\hat{\mathbf{r}})+\left[E_{1}^{\text {sca }}(\hat{\mathbf{r}})\right]^{*} E_{1}^{\mathrm{ref}}(\hat{\mathbf{r}})}{\left|E_{1}^{\text {ref }}(\hat{\mathbf{r}})\right|^{2}} .
\end{aligned}
$$

Often, the intensity of the reference wave at the detector is much greater than that of the scattered wave. This is especially true in this work where the objects being illuminated are small particles, as opposed to the macroscopic sized objects involved in other applications, see e.g. [13-16]. This means that the term $\left|E_{1}^{\text {sca }}(\mathbf{r})\right|^{2} /\left|E_{1}^{\text {ref }}(\mathbf{r})\right|^{2}$ in Eq. (4) can be neglected, leaving

$I^{\mathrm{con}}(\mathbf{r}) \simeq \frac{\left[E_{1}^{\mathrm{ref}}(\hat{\mathbf{r}})\right]^{*} E_{1}^{\mathrm{sca}}(\hat{\mathbf{r}})+\left[E_{1}^{\mathrm{sca}}(\hat{\mathbf{r}})\right]^{*} E_{1}^{\mathrm{ref}}(\hat{\mathbf{r}})}{\left|E_{1}^{\mathrm{ref}}(\mathbf{r})\right|^{2}}$.

This intensity pattern, which is the difference between two measurements - with and without the particle present - is known as a contrast hologram. The key characteristic of $I^{\text {con }}$ is its linear dependence on the amplitude of the particle's scattered wave. This means that the phase of the scattered wave over the detector is encoded in the measurement. Consequently, $I^{\text {con }}$ can be used to reconstruct unambiguously an image of the particle that closely resembles that obtained from conventional microscopy.

Because there are many references describing the theory behind digital holographic imaging, only a brief description will be given here, see e.g. [17-19]. Basically, the contrast hologram is envisioned as a transmission diffraction-grating illuminated by a normally incident plane wave, i.e., a reconstruction wave. The FresnelKirchhoff approximation is then used to describe the light diffracted from this grating in a parallel plane separated by a distance $z$ from the grating along the $z$-axis. If $z$ corresponds to the distance between the particle and detector during the hologram measurement $(z=d)$ the resulting diffraction pattern in this so-called reconstruction plane yields an image of the particle. The image is essentially equivalent to a conventional microscope image, although the resolution is typically less [3].

The advantage of using the Fresnel-Kirchhoff approximation to calculate the reconstructed particle image is that the approximation's mathematical form is essentially a discrete Fourier transform of the CCD pixel values constituting $I^{\text {con }}$. This enables the use of the fast Fourier transform (FFT) in the calculation, thus substantially reducing the computation time required to render the particle image. This is fortuitous, because in practice $d$ is not known to great enough accuracy to be able to reconstruct an image from a single application of the reconstruction routine. This inaccuracy is due to the variation in particle positions in the aerosol stream as they enter the measurement volume. Consequently, the image-reconstruction stage consists of a focusing-like procedure: First an initial image is reconstructed using an estimate of $d$ based on the experimental layout. Then, the reconstruction plane is scanned along the $z$-axis in 
small steps until the reconstructed image comes into focus. The ability to use the FFT for each of these intermediate steps is thus crucial to the practical implementation of this technique.

The primary drawback to the in-line configuration is that two images of the particle are produced in the reconstruction stage [17]. The in-focus particle image is always accompanied by a blurred twin image that is infocus in the mirror reconstruction plane, i.e., at $z=-d$. As a consequence, the image quality is degraded. However, as shown in [3], the effect of the twin on the in-focus image becomes negligible if both $d$ and the size of the CCD pixel array are sufficiently large such that an imaging resolution on the order of the wavelength can theoretically be achieved [3,4].

Another drawback of in-line holography is the presence of the zero frequency, or so-called DC, term in the reconstructed image [20]. In the diffraction-grating model, the reconstruction wave is uniform across the hologram since it is planar and normally incident. Upon application of the FFT to $I^{\text {con }}$, this wave then becomes a strong DC contribution in the transform. The result is an unwanted bright spot in the reconstructed image located at the intersection of the optical axis (z-axis) with the reconstruction plane. Fortunately, however, the DC term can be nearly eliminated by subtracting from each pixel value in $I^{\text {con }}$ the average value of all the pixels [17]. Notice that in doing this subtraction, the result is a new contrast hologram with both positive and negative values; whereas, its constituent holograms $I^{\text {holo }}, I^{\text {ref }}$, and $I^{\text {con }}$, are all inherently positive since they correspond to intensity measurements.

The resolution of the resulting particle images is limited by several factors related to diffraction and the apparatus hardware: the CCD pixel size, CCD pixel-array size $w$, particle-CCD distance $d$, and the illumination wavelength $\lambda[17,4]$. Given the configuration of the optical elements in this work, the theoretical minimum resolvable length scale is approximately $4 \mu \mathrm{m}$ following [4]. However, the resolution achieved in practice is in the range of $8-10 \mu \mathrm{m}$ due to stray-light noise and imperfections in the optical design. Fundamentally, the resolution of this holographic configuration will not exceed what is possible from a conventional optical microscope. However, as discussed earlier, it does provide the substantial advantage of near real-time, in situ, and high through-put imaging, which is not typically possible with conventional microscopy.

\section{Apparatus design and validation}

The experimental apparatus, which is shown in Fig. 1, consists of two primary subsystems: aerosol-particle sensing and hologram recording. An aerosol stream is delivered via a nozzle made from a plastic pipettor-tip to the measurement volume where an optical trigger is used to sense the presence of a particle [21,22]. This trigger consists of crossed diode-laser beams, labeled (h) and (i) in Fig. 1. These lasers have different wavelengths of 635 and $670 \mathrm{~nm}$ and intersect near the outlet nozzle delivering the aerosol. When a particle passes into this intersection it scatters both wavelengths of light simultaneously. The scattered light is received by two photomultiplier (PMT) modules (Hamamatsu Corp., model H6780-02), (j) in the figure, each sensitive to only one of the two wavelengths. A series of signal-analysis units determines if the signals produced by the PMT modules are coincident. If so, this indicates the presence of a particle at the trigger laser-beam intersection and a fire signal is sent to a pulsed laser for the hologram recording.

The triggered light source is a 70 ns pulsed Nd:YAG laser (Spectra Physics Lasers, Inc., model Y70-532Q), frequency doubled to $532 \mathrm{~nm}$. This light passes through a Glan-Thompson polarizer to ensure linear polarization (a) in Fig. 1. The light is then focused by lens (b) onto a $50 \mu \mathrm{m}$ diameter pinhole (c). Next the primary lobe of this pinhole diffraction pattern illuminates a second pinhole (d) with a diameter of $25 \mu \mathrm{m}$. These pinholes "clean" the beam improving its spatial coherence and enhancing the quality of the hologram. All but the primary lobe of this second pinhole pattern is blocked by iris (e) where lens (f) then collimates the beam, which is brought to a focus by lens $(\mathrm{g})$ at a point approximately $2 \mathrm{~mm}$ from the aerosol nozzle outlet. This $2 \mathrm{~mm}$ is the distance $l$ in Section 2. In this way, the aerosol particles are illuminated by what is approximately a spherical wave originating from the beam waist. The beam continues until reaching the $C C D$ detector (Finger Lakes Instrumentation, LLC, model ML8300), at which point it expands to fill the entire pixel array $(5.4 \mu \mathrm{m}$ pixel size, $3326 \times 2504$ pixel-array size) The separation between the particle stream and the detector is the $d$ discussed in Section 2 and is approximately $8 \mathrm{~cm}$. A small amount of the beam is scattered by the particle (dashed line in Fig. 1), and this light interferes with the remainder of the beam, i.e., the reference wave, to form the interference pattern that becomes the digital hologram $I^{\text {holo }}$.

To test the apparatus and provide a rough calibration of the image-reconstruction procedure, a comparison is made between a holographic and optical microscope image of the same particle. This is done by placing $15.4 \mu \mathrm{m}$ diameter NIST-traceable polystyrene latex microspheres (Duke Scientific Corp.) on a microscope slide and positioning the slide in the measurement volume at the intersection of the trigger-beams. A hologram is recorded, from which the image-reconstruction procedure of Section 2 is followed. The slide is then transferred to a microscope, where the same spheres are located and imaged. Next, using a 1951 USAF glass-slide resolution target (Edmund Optics), a scale factor is determined relating the microscope-image pixel number to micrometers. Then, by comparing the holographic image of a microsphere to the microscope image of the same microsphere, an additional scale factor is determined relating the hologram pixel number to micrometers. In this way, the holographic images of all subsequent particles can be rendered in calibrated length (micrometers), rather than pixel number. This calibration procedure is approximate, however, because there is ambiguity in determining the hologram pixel-number size of a given microsphere: The contrast between the reconstructed sphere-image and the background is not sharp, which 

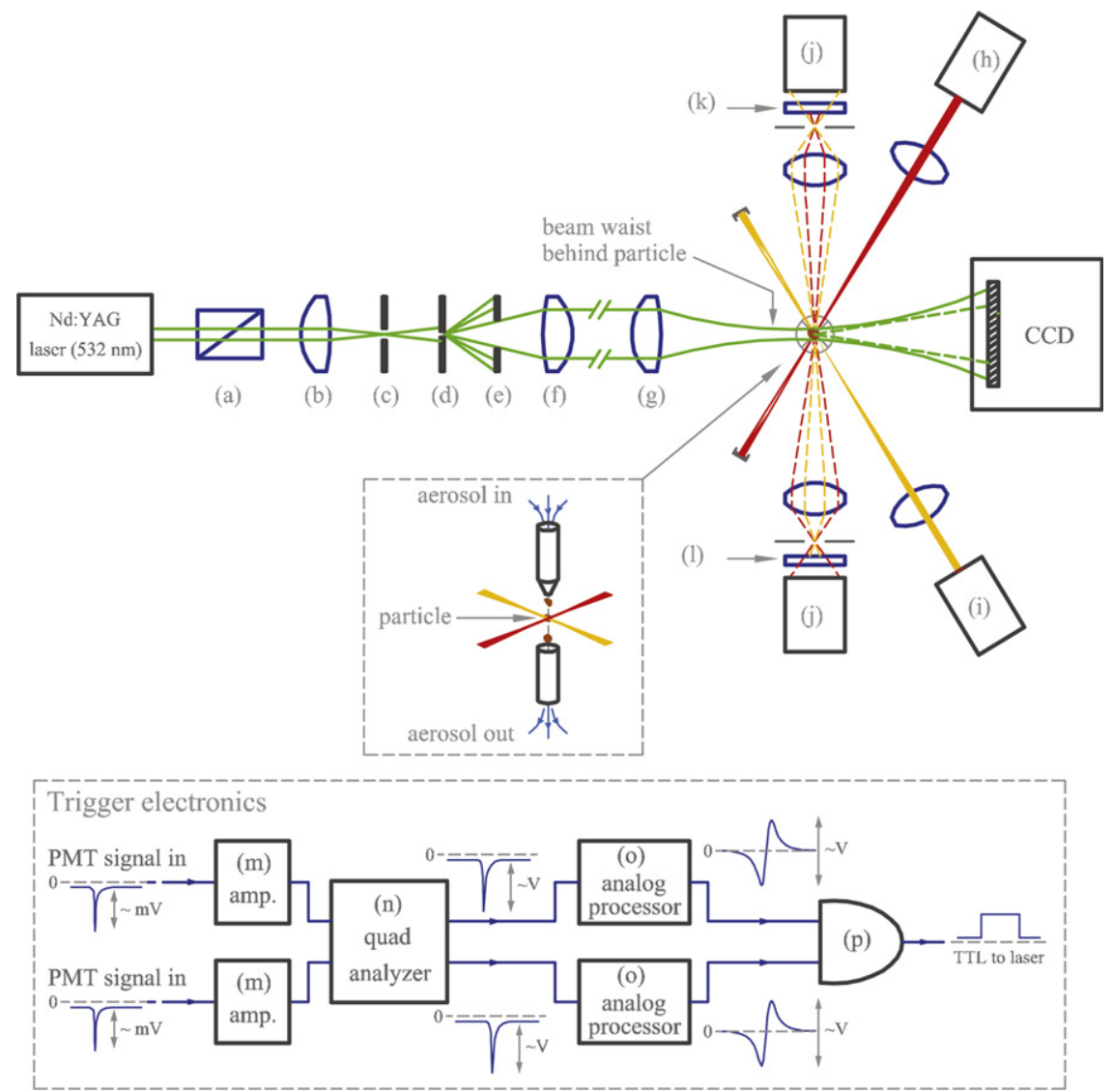
(a) Glan-Thompson polarizer
(b) lens, $\mathrm{f}=75 \mathrm{~mm}$
(c) 50 um pinhole
(d) 25 um pinhole
(e) blocking iris
(f) lens, $\mathrm{f}=300 \mathrm{~mm}$

(g) lens, $\mathrm{f}=30 \mathrm{~mm}$

(m) ORTEC Mo. 570 amplifer

(h) diode laser $670 \mathrm{~nm}$

(i) diode laser $635 \mathrm{~nm}$

(n) ORTEC Mo. 850

(j) photomultipler mod.

(o) SRS analog processor

(k) $635 \mathrm{~nm}$ line filter

(l) $670 \mathrm{~nm}$ line filter

(p) ORTEC Mo. CO4020 AND

Fig. 1. Diagram of the apparatus. The middle inset shows a schematic of the signal-analysis electronics used in the optical trigger to sense the presence of a particle in the measurement volume. See text for further explanation.

can yield a scale factor that over- or under-determines the particle size.

An example is presented in Fig. 2 demonstrating the comparison between the holographic and microscope images of the same particle. Here a cluster of ragweed pollen particles is placed on a microscope slide, then holographic and microscope images of the cluster are obtained. By comparing these images, one can see that the holographic apparatus successfully produces an accurate image of the pollen cluster, with sufficient resolution to discern individual pollen particles and even a faint signature of the single-particle surface roughness seen in the microscope images. This corresponds to a resolution roughly between $8-10 \mu \mathrm{m}$, although a more rigorous resolution analysis is not performed. Referring to the measured and contrast holograms shown in this figure, one can see how subtraction of the incident beam across the $\mathrm{CCD}$, i.e., $I^{\text {ref }}$, removes noise due to imperfections in the incident beam profile. This has the consequence of producing a "cleaner" contrast hologram, which subsequently improves the particle image. Note that the holographic and microscope images of the cluster differ slightly in overall size and detailed structural form. Although it is clearly the same cluster in (c) and (d), the differences are likely due to shifting of the cluster on the microscope slide during transfer from the apparatus to the microscope.

There are several unique aspects to the design of this apparatus. By using the short focal-length lens (g) in Fig. 1 to form a beam waist near the particle, the light illuminating the particle is more intense than it would be if only the pinhole was used for illumination (as is usually done). This results in a relative amplification of the scattered wave at the detector and enhances the interference structure of the hologram leading to improved particle-image quality. Using a pulsed laser permits the 
a

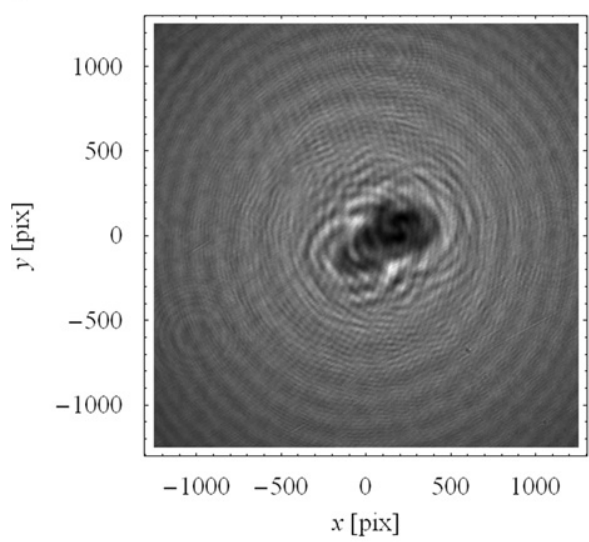

C

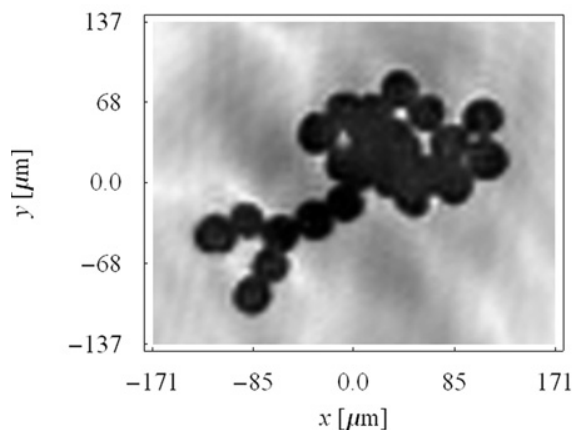

b

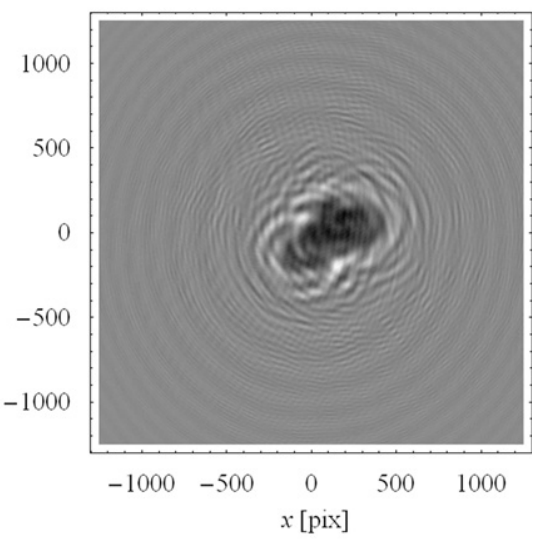

d

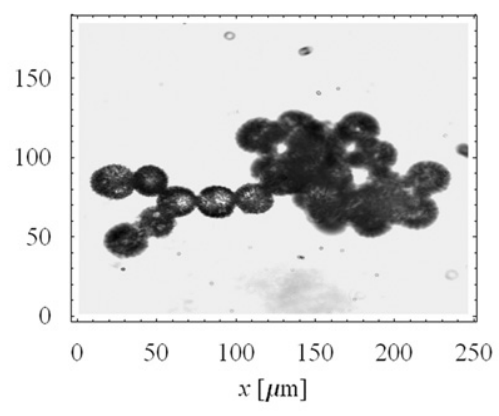

Fig. 2. Validation of the holographic imaging apparatus. Plots (a) and (b) show the measured $I^{\text {holo }}$ (digital) and corresponding contrast $I^{\text {con }}$ holograms, respectively, for a cluster of ragweed pollen particles on a microscope slide located at the intersection of the trigger-beams, recall Fig. 1. Image (c) shows the reconstructed image resulting from (b) whereas (d) shows a conventional microscope image of the same cluster.

investigation of particle systems in motion. This also greatly relaxes the strict mechanical-stability demands typically required for holographic measurements. There are no optical elements between the aerosol stream and the CCD. This gives the apparatus a working distance of several centimeters, which is substantially greater than the single- to sub-millimeter working distance of a microscope objective. Moreover, the absence of any optical elements between the detector and particle eliminates "noise" resulting from ambient dust that can collect on the optical surfaces.

\section{Applications}

To further assess the imaging capabilities of the apparatus, several aerosols consisting of complex-shaped particles are examined. The first samples are sieved Saharan and Tunisian sand, which are aerosolized using an Erlenmeyer flask as follows: A small sample of the sand is placed in the flask, then sealed with a stopper. Two aluminum tubes pass through the stopper; one supplies air to the flask, blowing the sand particles around, while the other tube allows some of the airborne particles to exit the flask and be transported to the aerosol nozzle in the apparatus. Fig. 3 shows the contrast holograms along with the resulting particle-image reconstructions for single
Saharan and Tunisian sand particles. For comparison, Fig. 4 shows microscope images of these sand samples. One can see that the holographic images provide the same information of overall particle size and morphology as the microscope images. For example, the Saharan particles appear to have less surface roughness than the Tunisian particles. Note that unlike Fig. 2, the particles shown in the holographic reconstructions (Fig. 3) and microscope images (Fig. 4) are not the same sand particles since the holographic images are obtained from flowing particles.

Another unique capability of holographic imaging is that some sense of the three-dimensional form of a particle can be garnered from a single measurement. The basic idea is analogous to the "focusing in" on a particle in conventional microscopy. There, the microscope objective is moved vertically to vary the distance between it and the microscope slide, causing a blurred image of a particle to evolve into a sharp image. If the particle has sufficient thickness and transparence, different depths within the particle can be brought into focus to give a feel for the particle's three-dimensional structure. This same process can be done in digital holography by computationally varying the distance $d$ used in the imagereconstruction stage, as is shown in [3]. The resulting sequence of images gives the same impression of focusing in on the particle as one gets from microscopy. However, 
a

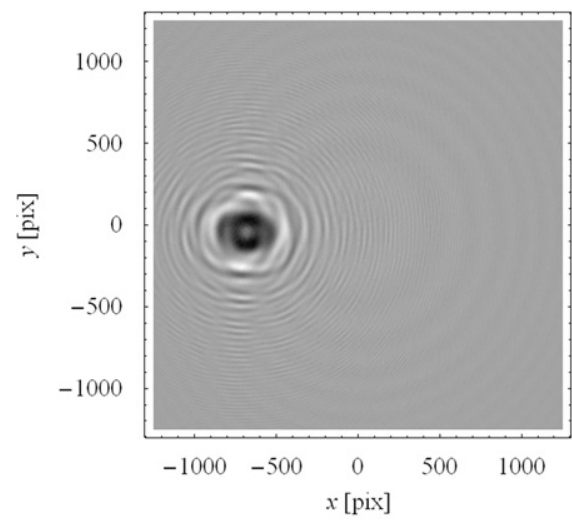

C

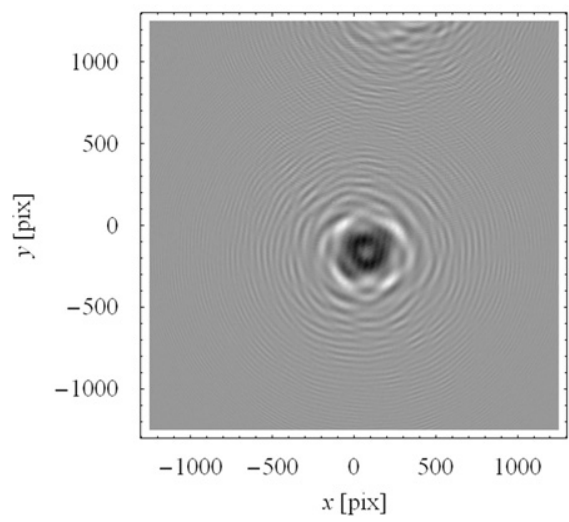

b

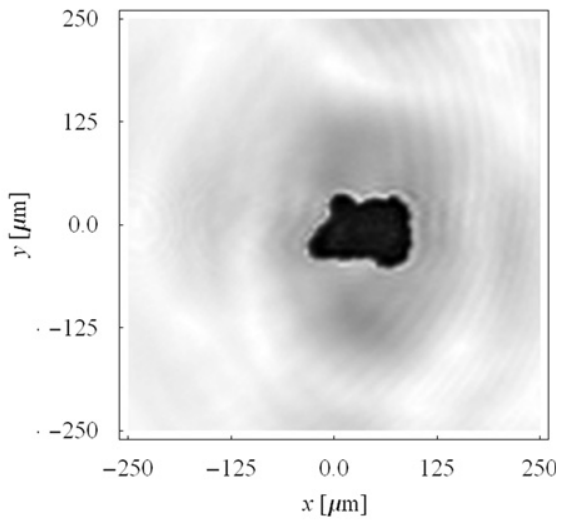

d

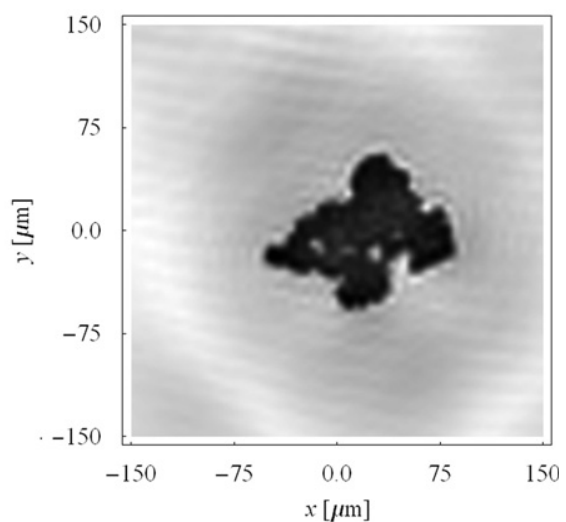

Fig. 3. Saharan and Tunisian sand particles. Images (a) and (b) show the contrast hologram $I^{\text {con }}$ and corresponding reconstructed image for a single Saharan sand particle. Images (c) and (d) show the same for a single Tunisian sand particle.

a

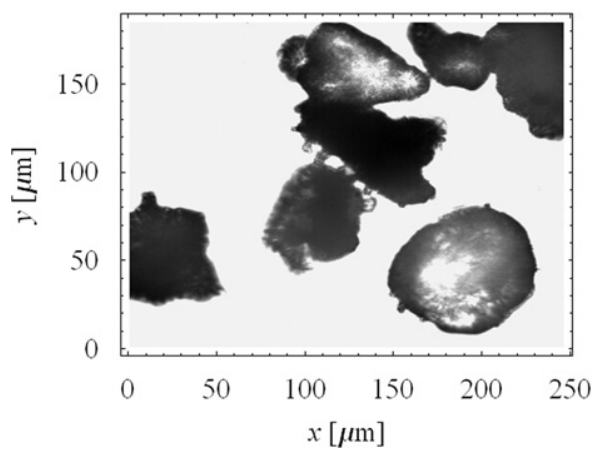

b

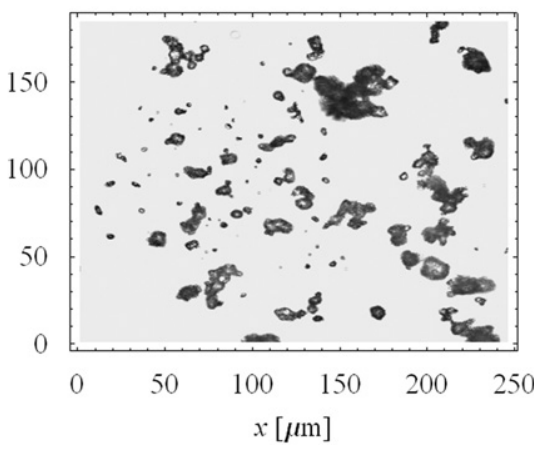

Fig. 4. Microscope images of (a) Saharan and (b) Tunisian sand. The particles seen here are taken from the same sand samples used in Fig. 3, but unlike the ragweed in Fig. 2, these particles are not the exact same particles imaged holographically.

unlike microscopy where an image must be recorded at each "focus depth," the holographic route can obtain a similar image-sequence from the (single) contrast hologram only.

Fig. 5 shows an example of this holographic focusing process. The top row displays conventional microscope images of a $\mathrm{NaCl}$ crystal at different focus depths. The bottom row shows a holographic image-sequence for an aerosolized $\mathrm{NaCl}$ particle that is produced by scanning the reconstruction plane along the $z$-axis around $z=d$. The particle in the holographic images is delivered to the apparatus in aerosol form by drying a salt solution on a hotplate and aerosolizing the resulting powder using the Erlenmeyer generator described in Section 3. One can 
a
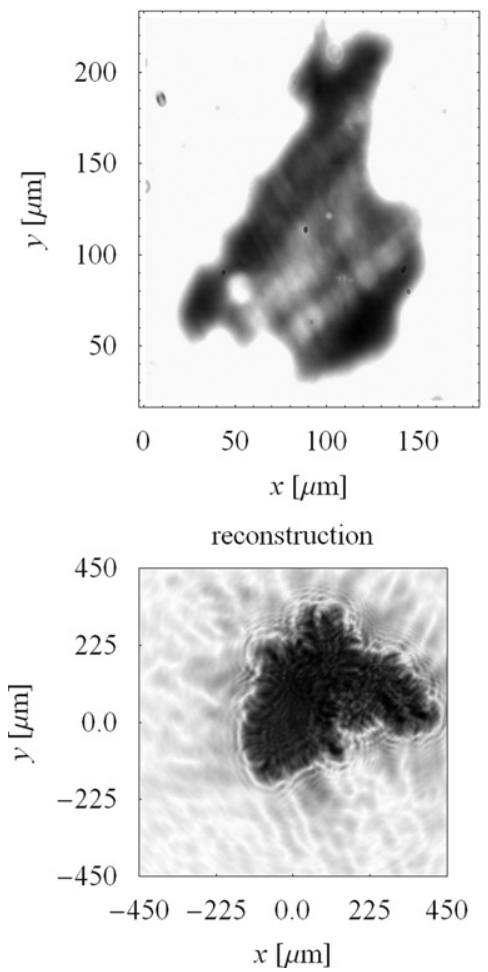

b
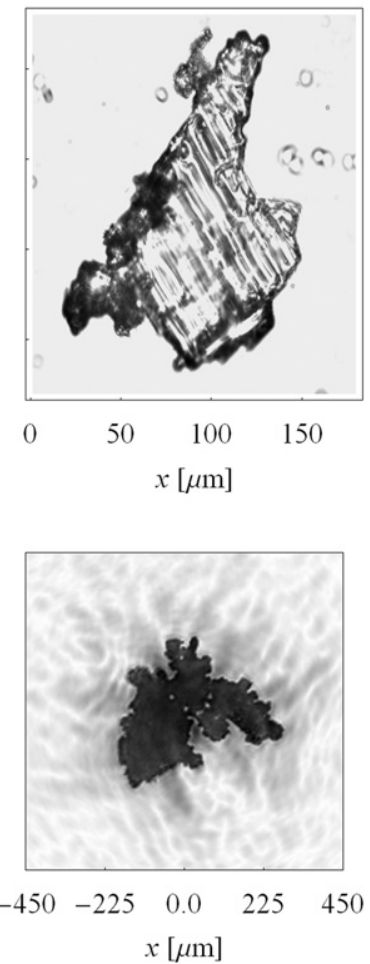

C
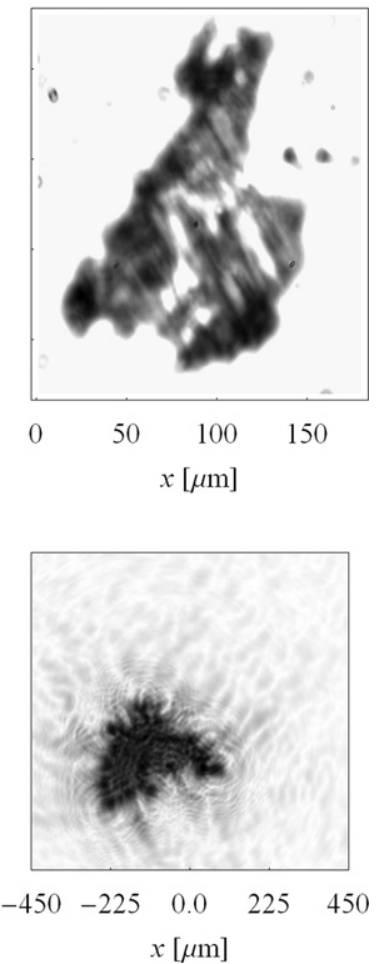

Fig. 5. Focusing behavior of the holographic image-reconstruction process. The top row shows microscope images of a NaCl crystal on a microscope slide at three different focus depths (a)-(c). The bottom row shows the reconstructed images of a NaCl aerosol particle when the reconstruction plane is at three positions for $z$ : $z<d$ for (a), $z=d$ for (b), i.e., in-focus, and $z>d$ for (c).

clearly see the strong similarity in the focusing behavior of the two imaging techniques.

\section{Comments}

The in situ images of aerosol particles presented here are not the only documented examples. Sorensen et al. have obtained images of the particles constituting hydrocarbon-flame soot at various stages in soot formation, i.e., as a function of height in a flame [23,24]. Here a $10 \times-$ power photomicroscope is mated to a conventional filmcamera and a $1.5 \mu$ s Xe flash lamp is used for particle illumination. With this arrangement, particles in the range of roughly $5-100 \mu \mathrm{m}$ are imaged, which covers the same particle size range considered in our work. One might then wonder what advantage the holographic approach offers over this photomicroscope direct-imaging.

First, the photomicroscope images are obtained photographically, i.e., using film, requiring chemical processing. The holograms, however, are entirely digitally recorded and the resulting images are computationally rendered. Second, and perhaps most important, the photomicroscope images have a very narrow depth of field, and only particles constrained within a narrow volume are infocus; whereas, for holographic techniques the focusing is done computationally, after the hologram is recorded. This enables the focusing process described in Section 4, which can be used to image multiple particles present at different locations in the measurement volume as demonstrated in [3]. Moreover, this can be done from a single hologram recording. To do this with the photomicroscope would require obtaining a series of exposures with the microscope objective positioned at different distances from the measurement volume. Thus, if the particles are in motion, as they are in flow-through applications, a series of exposures would prevent the imaging of multiple particles present at a given instant in the measurement volume.

As mentioned in Section 3, an inherent advantage of the holographic design is that there are no optical elements between the particle and detector. Thus, there are no surfaces for ambient dust to collect on and become sources of stray light, nor are there any lens-based aberrations and multiple reflections. Both of these concerns are present in the photomicroscope approach. The absence of these optical elements in the holographic design is especially advantageous when one wishes to investigate particles that are roughly the same size as ambient dust.

\section{Conclusion}

This work demonstrates the feasibility of imaging single and multiple aerosol particles in situ using digital in-line holography. Imaging is demonstrated on ragweed pollen, Saharan and Tunisian sand, and $\mathrm{NaCl}$ particles; a 
range of overall particle-size covering approximately $15-500 \mu \mathrm{m}$. These images are computationally reconstructed from the digitally recorded holograms and compare well to the corresponding microscope images. Although the resolution of the holographic images is less than those from the microscope, one is able to clearly discern single-particle size and shape. Moreover, the ability to computationally render the images allows the application of numerical operations to improve image quality, whereas the analogs of such operations in conventional optical imaging would be difficult to implement.

\section{Acknowledgments}

This work was supported by the Defense Threat Reduction Agency and the US Army Research Laboratory. We are thankful for helpful discussions with Yong-Le Pan, Steve Hill, Chris Sorensen, Dave Ligon, and Jay Eversole.

\section{References}

[1] Aptowicz KB, Pinnick RG, Hill SC, Pan YL, Chang RK. Optical scattering patterns from single urban aerosol particles at Adelphi, Maryland, USA: a classification relating to particle morphologies. J. Geophys. Res. 2006;111:D12212.

[2] Hoekstra A, Maltsev V, Videen G, editors. Optics of Biological Particles. Dordrecht: Springer; 2007.

[3] Xu W, Jericho MH, Meinertzhagen IA, Kreuzer HJ. Digital in-line holography of microspheres. Appl. Opt. 2002;41:5367-75.

[4] Garcia-Sucerquia J, Xu W, Jericho SK, Klages P, Jericho MH, Kreuzer HJ. Digital in-line holographic microscopy. Appl. Opt. 2006;45:836-50.

[5] Kreuzer HJ, Jericho MJ, Meinertzhagen IA, Xu W. Digital in-line holography with photons and electrons. J. Phys. Condens. Matter 2001;13:10729-41.

[6] Raupach SMF, Vossing HJ, Curtius J, Borrmann S. Digital crossedbeam holography for in situ imaging of atmospheric ice particles. J. Opt. A: Pure Appl. Opt. 2006;8:796-806.

[7] Fugal JP, Shaw RA. Cloud particle size distributions measured with an airborne digital in-line holographic instrument. Atmos. Meas. Tech. 2009;2:259-71.
[8] Singh VR, Hegde G, Asundi A. Particle field imaging using digital inline holography. Curr. Sci. 2009;96:391-7.

[9] Thompson BJ. Holographic particle sizing techniques. J. Phys. E: Sci. Instrum. 1974;7:781-8.

[10] Bogan MJ, Starodub D, Hampton CY, Sierra RG. Single-particle coherent diffractive imaging with a soft X-ray free electron laser: towards soot aerosol morphology. J. Phys. B: At. Mol. Opt. Phys. 2010;43:1-13.

[11] Bogan MJ, Boutet S, Chapman HN, Marchesini S, Barty A, Benner WH, et al. Aerosol imaging with a soft X-ray free electron laser. Aero. Sci. Tech. 2010:44:i-iv.

[12] Mishchenko MI, Travis LD, Lacis AA. Multiple scattering of light by particles: radiative transfer and coherent backscattering. Cambridge: Cambridge University Press; 2006.

[13] Schnars U, Juptner W. Direct recording of holograms by a CCD target and numerical reconstruction. Appl. Opt. 1994;33:179-81.

[14] Zhang S. Application of super-resolution image reconstruction to digital holography. EURASIP J. Appl. Signal Process. 2006: 1-7 [article ID 90358].

[15] Ma L, Wang H, Li Y, Jin H. Numerical reconstruction of digital holograms for three-dimensional shape measurement. J. Opt. A: Pure Appl. Opt. 2004;6:396-400.

[16] Javidi B, Kim D. Three-dimensional-object recognition by use of single-exposure on-axis digital holography. Opt. Lett. 2005;30: 236-8.

[17] Kreis T. Handbook of holographic interferometry, optical and digital methods. Germany: Wiley; 2005.

[18] Kim MK. Principles and techniques of digital holographic microscopy. SPIE Rev. 2010;1:018005-1-50.

[19] Ferraro P, De Nicola S, Coppola G. Digital holography: recent advancement and prospective improvements for applications in microscopy. In: Optical imaging sensors and systems for homeland security applications, Advanced Sciences and Technologies for Security Applications, vol. 2; 2006. p. 47-84.

[20] Goodman JW. Introduction to Fourier optics. Englewood: Roberts \& Company; 2005.

[21] Pan YL, Holler S, Chang RK, Hill SC, Pinnick RG, Niles S, et al. Singleshot fluorescence spectra of individual micrometer-sized bioaerosols illuminated by a 351- or 266-nm ultraviolet laser. Opt. Lett. 1999;24:116-8.

[22] Berg MJ, Hill SC, Pan Y-L, Videen G. Two-dimensional Guinier analysis: application to single aerosol particles in-flight. Opt. Ex. 2010;18:23343-52.

[23] Sorensen CM, Hageman WB. Two dimensional soot. Langmuir $2001 ; 17: 5431-4$

[24] Sorensen CM, Hageman WB, Rush TJ, Huang H, Oh C. Aerogelation in a flame soot aerosol. Phys. Rev. Lett. 1998;80:1782-5. 